\title{
RADIATION STUDIES IN THE ANTIPROTON SOURCE
}

\author{
Mike Church 6/10/91
}

\begin{abstract}
Experiment $\mathrm{E} 760$ has a lead $\mathrm{glass}(\mathrm{Pb}-\mathrm{G})$ calorimeter situated in the antiproton source tunnel in the accumulator $r i n g$ at location $\mathrm{A} 50$. This location is exposed to radiation from several sources during antiproton stacking operations. A series of radiation studies has been performed over the last two years to determine the sources of this radiation and as a result, some shielding has been installed in the antiproton source in order to protect the lead glass from radiation damage.
\end{abstract}

\section{MOTIVATION}

The E760 Pb-G calorimeter contains 1280 pieces of lead glass of 20 different shapes $40-50 \mathrm{~cm}$ long and each weighing approximately $10 \mathrm{~kg}$. The total cost of the lead glass, including machining, exceeds $\$ 1,000,000$. Each block is instrumented with a photomultiplier and $A D C$, and the digitized charge from the $A D C$ is used to determine electromagnetic energy deposited in the lead glass -- hence the calibration and stability of these signals are very important. It is well known that lead glass suffers damage (loss of light transmission) from radiation. (1) Our own measurements show that a dose of $300-400$ rads will reduce light transmission about $50 \%$ in a $50 \mathrm{~cm}$ long piece of F2 glass similar to that installed in the $E 760$ calorimeter. (2) The studies reported here were conducted to determine how much radiation is present at $A 50$ (location of the calorimeter) during antiproton stacking, what its sources are, and what can be done to reduce it.

Figure 1 is a plan view of the antiproton source. During stacking operations, a beam of 9 Gev negative particles (momentum spread about 4\%) is injected from the AP-2 I ine into the debuncher ring on '29' cycles, circulated for about 2 seconds, and then transferred to the accumulator ring where antiprotons are "stacked". The ratio of pions to antiprotons at the end of the AP-2 I ine is about 100/1.(3) These pions all decay in the debuncher and only antiprotons are transferred to the accumulator. A pulse of beam about 1 nsec in duration is delivered down AP-2 about once every 3 seconds, although this rate will vary depending upon other accelerator operations. A typical good antiproton stacking rate is $1 \mathrm{~mA} / \mathrm{hr}(10 * * 10$ antiprotons/hr); thus about $10 * * 12$ pions/hr are delivered to the antiproton source

1) "Radiation on F2 Lead Glass and Consequences for the Monitoring of the Absolute Calibration of Lead Glass Calorimeters" Kirsebom and Sollie, Nuclear Instruments and Methods, A245 (1986), p. 351-360

2) J Marques, E760 internal memo, Nov. 1989

3) private communication, J. Marriner 


\section{MEASUREMENT TECHNIQUE}

All radiation measurements were made using the standard radiation monitors deployed around the lab ("chipmunks"). These are propane filled ionization chambers in which charge from ionizing particles is collected and integrated (with a 3 or 10 second time constant). The unit outputs a TL level pulse for each $2.5 * 10 * *-6$ rads detected. These pulses were then scaled and the scalers read out with the accelerator division ACNET controls system. The scalers are reset once every supercycle and the average over several supercycles is recorded. All monitors were calibrated by the Fermilab safety division before being used for these studies. Each unit has an internal source which generates a few counts per minute, but this was ignored since the typical count rate observed in these studies ranged from a few hundred to a few thousand. For these counters, 1 rad corresponds to $5.1 * 10 * * 11$ minimum ionizing particles per meter**2 which is 400,000 counts.

We attempted to use loss monitors designed for use in the Tevatron to measure the dose rates in the AP-2 line. These consisted of a can of liquid scinitillator instrumented with a photomultiplier and charge integrator. They had a large background rate (noise and DC offset at the input of the $A D C$ ) and could not accurately measure the low radiation rates observed.

\section{INITIAL MEASUREMENTS $6 / 89$}

A 1st series of studies were conducted in June of 1989 during antiproton stacking operations before the $\mathrm{Pb}-\mathrm{G}$ calorimeter was installed.

1) Monitors were placed in the locations shown in Figure 2; no shielding blocks were installed.

a) The pulsed magnet which steers beam onto the antiproton production target was turned off, preventing any beam from entering the AP-2 line and leaving only circulating beam in the accumulator. All dose rates went to 0 . This demonstrates that circulating beam in the accumulator is not a source of radiation at $A 50$.

b) The bend magnets halfway down the AP-2 I ine were turned off, preventing any beam from entering the downstream end of AP-2. All dose rates went to 0 . This demonstrates that the upstream end of $A P-2$ is not a source of radiation at $A 50$.

c) The monitor D3 near the debuncher but well upstream of the AP-2 line and the injection region showed a dose rate of about $.01 \mathrm{rads} / \mathrm{hr}$.

This indicates that circulating beam in the debuncher is not a significant source of radiation at $A 50$.

d) The vertical bend in AP-2 (IBV1) was turned off, preventing any beam from being injected into the debuncher. The radiation at $A 50$ decreased, which means that at least some of the radiation comes from the injection area of the debuncher. 
e) A $1 / 2^{n}$ aluminum plate was inserted into an air gap in the AP-2 beamline downstream of Q28 (see Figure 5). Radiation at A50 increased by only $75 \%$, demonstrating that the air gap in the AP-2 beamline is not a significant source of radiation at $A 50$.

The general conclusion from measurements a)-e) is that the radiation at $A 50$ has 2 possible sources: the injection section of the debuncher and the downstream end of the AP-2 line.

2) Collimators in the upstream end of the AP-2 line were adjusted in an attempt to minimize the dose rates at A50. At this time, 2 sets of vertical collimators and 2 sets of horizontal collimators were used. A group of "optimum" settings were found which reduced radiation at $A 50$ by a factor of 6.5 while reducing circulating debuncher beam by only $20 \%$ (see Figure 2).

3) 13 movable shielding blocks consisting of steel cages filled with cinder blocks and steel plates were used to try and shield radiation from the injection section of the debuncher. These cages are $32^{\prime \prime} \times 25^{\prime \prime} \times 55^{\prime \prime} h$ igh, and the steel plates occupy a volume of $16^{n}$ deep $\times 25^{n} \times 24^{n}$ centered at beam height for a total of 4.8 interaction lengths at a 45 degree angle of incidence. 7 different configurations were tried and radiation doses were measured after each configuration. Figures 3a - 3d show 4 consecutive configurations. The conclusions drawn from this study are:

a) the debuncher kicker is a large source of radiation;

b) the debuncher septum is also a significant source of radiation;

c) no other sources of radiation were found; and

d) local shielding reduced the dose at $A 50$ by a factor of 1.7 with collimators in optimum position (compare Figure 3a with Figure 2 normalized to 19 ' 29 ' cycles).

4) 10 concrete shielding blocks, $35^{n} \times 35^{n} \times 72^{n}$, were placed between the debuncher and accumulator upstream of $A 50$ in an attempt to block radiation coming from AP-2. No change in dose rate was measured at $A 50$. Since this shielding is $5 * 35 / 16=11$ interactions lengths, we conclude that the radiation coming from $A P-2$ is primarily muons. $d E / d x=3.7 \mathrm{Mev} / \mathrm{cm}$ for concrete, so that $5 * 35^{\prime \prime}$ can only effectively shield muons with energy less than about $2 \mathrm{Gev}$. 
The production of muons from primary ( $9 \mathrm{Gev}$ ) decaying pions was simulated with a version of "Decay Turtle"(4) in order to investigate this as a possible source of radiation at A50. Decay Turtle is a monte carlo program which tracks pions and their daughter particles (muons and neutrinos) through beamline elements. Decay Turtle was 1 st run tracking only pions to verify reasonable agreement with actual SEM displays from the AP2 line. (5) Initial phase space distributions were chosen broad enough to $f i l l$ the acceptance of the downstream end of AP-2. Additional code was added to track muons outside the beampipe aperture with multiple scattering in steel (bends, trims, and quads) and bending in quadrupole steel simulated. An accurate field map of the quadrupoles was used. The final result is shown in the scatter plot of Figure 4 . Each entry in the scatter plot represents 1 muon, and the 1800 entries in the plot correspond to 9200 pions at the end of AP-2, or 92 antiprotons. To normalize this to $1 \mathrm{~mA} / \mathrm{hr}$ of stacking (assuming $80 \%$ debuncher-to-accumulator efficiency), each entry/m**2 in the scatter plot represents $10 * * 10 / 92 / .80=1.4 * 10 * * 8$ min imum ionizing particles/m**2/hr or $1.4 * 10 * * 8 / 5.1 * 10 * * 1=3 * 10 * *-4 \mathrm{rads} / \mathrm{hr}$. The plot has approximately 1 entry in the square meter centered on the accumulator which represents a dose rate 200 times smaller than the observed dose rate.

The conclusion is that muons from PRIMARY decaying pions is not a significant source of radiation at $A 50$.

\section{MEASUREMENTS $2 / 90$ TO $9 / 90$}

Some of the radiation studies of 6/89 were repeated between 2/90 and $4 / 90$ with the results shown in the following table. Where noted, the shielding was installed in a configuration very similar to that shown in Figure 5. The results agree fairly well with the measurements done on $6 / 89$ with the exception that the collimators are not as effective as they were on $6 / 89$ with collimators in "optimum" position.

$\begin{array}{llll}\text { DATE '29' CYCLES/ } & \text { DOSE a A50 } & \text { D:FFTTOT (nA) } \\ \text { SUPERCYCLE } & \text { SHIELDING } & \text { COLLIMATORS } \\ \text { debuncher beam }\end{array}$

$\begin{array}{lrrrrr}2 / 25 & 20 & 1081 & 1150 & \text { NO } & \text { OUT } \\ 2 / 25 & 20 & 212 & 900 & \text { NO } & \text { OPTIMUM } \\ 3 / 17 & 18 & 1108 & 1800 & \text { YES } & \text { OUT } \\ 3 / 17 & 18 & 280 & 1100 & \text { YES } & \text { OPTIMUM } \\ 4 / 1 & 4 & 267 & 1700 & \text { YES } & \text { OUT } \\ 4 / 1 & 4 & 73 & 1000 & \text { YES } & \text { OPTIMUM }\end{array}$

Data for the measurements done on $4 / 1 / 90$ are shown in Figure 5 as circled numbers in rads/hr normalized to 19 ' 29 ' cycles/supercycle.

4) "A Computer Program for Simulating Charged Particle Beam Transport Systems, Including Decay Calculations", SLAC-246 UC-28, March 1982

5) Antiproton Source Beamline Operations Manual

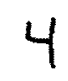


During the accelerator shutdown between $4 / 90$ and $6 / 90$, the AP-2 I ine was modified (bending magnet apertures opened up and a toroid removed from the

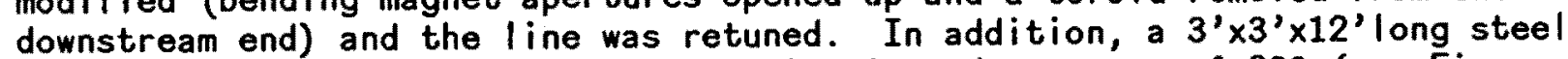
shield was placed around the AP2 beampipe just downstream of Q26 (see Figure 5) under the hypothesis that the radiation at $A 50$ was due to muons from secondary pions--that is, primary pions leaving the beampipe, interacting in the quadrupole steel, and producing secondary pions which then decay to muons. If this were the case, the 12' of steel would effectively stop pions which had exited the beam pipe.

During the 1st E760 phase 2 run (lead glass calorimeter installed) from $6 / 90$ to $9 / 90$ a survey of dose rates in the AP2 tunnel was made and these numbers are shown in Figure 5 ? In addition, radiation at $A 50$ was continually monitored and the AP2 collimators were re-optimized. It was found that:

1) Radiation is present throughout the downstream end of the AP2 tunnel. The observed rates are consistent with the rates observed at $A 50$.

2) There is evidence that Q27 is a source of radiation.

3) The $3^{\prime} \times 3^{\prime} \times 12^{\prime}$ steel shield was ineffective in reducing radiation.

4) Radiation rates at A50 were somewhat higher than previously measured (.08 rads/hr at the edge of the E760 pit).

5) The AP-2 collimators were not nearly as effective as they had previously been. Putting the collimators in "optimized" position reduced the radiation at $A 50$ a factor of 3.5 while reducing the stacking rate by $50 \%$.

6) During the course of the run the $\mathrm{Pb}-\mathrm{G}$ calorimeter absorbed a total of 70 rads of radiation as measured at the edge of the E760 pit. A flashlamp monitoring system on the $\mathrm{Pb}-\mathrm{G}$ shows a gradual loss of signal (6\%) in the upper half of the calorimeter but not in the the bottom half of the calorimeter. It is not clear yet if radiation is a cause of this loss in transmission.

7) $1435^{n} \times 35^{n} \times 72^{n}$ high concrete blocks were placed between the accumulator and debuncher just upstream of $A 50$ for the last month of the run. As in $6 / 89$, this had no effect on the radiation rates measured at $A 50$.

MEASUREMENTS $12 / 90-1 / 91$

Under the assumption that most of the radiation at $A 50$ was in the form of muons coming down the AP-2 enclosure, more shielding was added as shown in Fig. 6 . in 11/90. Measurements made during stacking operations from $12 / 90$ to $1 / 91$ showed no measurable reduction in radiation doses at A50 from the previous summer. Extensive tuning of AP-2 increased the efficiency of the AP-2 line (D:FFTTOT/M:TOR109) but did not decrease the radiation at $A 50$ relative to beam on target $(A: 760 R A D)$. It was found that if the Debuncher injection kicker timing was not set correctly (to within +-50 nsec) the radiation at $A 50$ would increase significantly. In addition, variations in dose rates as a function of location in the vicinity of A50 indicated that there were significant local sources of radiation in the injection section of the Debuncher from D4Q5 to DRF1-3 and that shielding there was probably not adequate. 
The collimators in the upstream end of AP-2 were moved and "optimum" settings were found which diminished radiation at $A 50$ by a factor of 3.6 while reducing the stacking rate by a factor of 2 -- conditions similar to stacking operations during the Summer of 1990.

The radiation was reverified as being "prompt": that is, it all occurs during injection and not during subsequent Debuncher or Accumulator turns. In addition, the short duration time structure of the radiation, as determined by viewing the E760 scintillation counter response, is very similar to the beam structure in the 1st turn of the Debuncher, as determined from the Deburicher gap monitor. The ${ }^{-84}$ bunch structure within -1.5 usecs is eviderit, with no long term or slow component evident. However, if some part of the Accumulator stochastic cooling system fails so that the stacking rate drops noticeably, an increase in radiation at $A 50$ is observed.

It is clear from these observations that the source of radiation at A50 is still not understood, however in an effort to further reduce this problem additional shielding was installed in $2 / 91$ as shown in Fig. 7 . The effect of this shielding remains to be tested during pbar operations from 6/91 to 11/91. It should be pointed out that the nature of the radiation is also not known (minimum ionizing, fast neutrons, slow neutrons...?). The radiation monitors are calibrated under the assumption that the radiation is all fast neutrons. If in fact the radiation is entirely minimum ionizing, then the actual radiation dose is 10 times lower than what is being claimed in this memo. If the radiation is primarily slow neutrons, than the actual dose could be substantially higher than what is reported here. However, given that there is a large prompt signal in the E760 scintillators, leads me to believe that the radiation is not primarily slow neutrons.

\section{CONCLUSIONS}

Current radiation rates at $A 50$ have the potential to damage the E760 lead glass calorimeter during the next fixed target run, 6/91 - 11/91. Therefore radiation doses at $A 50$ must be carefully monitored and kept to a minimum and the $\mathrm{Pb}$ glass transmission must also be carefully monitored to assess damage. The sources of the radiation and the nature of the radiation are still not completely understood. Shielding has already greatly mitigated the problem. 
APPENDIX

$1 \mathrm{rad}=6.24 * 10 * * 10 \mathrm{Mev} / \mathrm{kg}$ deposited energy.

If $N=$ density of minimum ionizing particles (particles/cm**2), then $R=(N / 6.24 * 10 * * 7) *(d E / d x) / d)$, where $R$ is dose in rads $\mathrm{dE} / \mathrm{dx}$ is energy loss in Mev/cm $d$ is density in $\mathrm{g} / \mathrm{cm} * * 3$.

For propane filled radiation monitors, $\mathrm{dE} / \mathrm{dx}=2.57 * 10 * *-3 \mathrm{Mev} / \mathrm{cm}$ $d=2.1 * 10 * *-3 \mathrm{~g} / \mathrm{cm} * * 3$,

therefore $N=R * 5.1 * 10 * * 7$.

For $\mathrm{Pb}-\mathrm{G} \mathrm{dE} / \mathrm{dx}=5.4 \mathrm{Mev} / \mathrm{cm}$ and $\mathrm{d}=3.61 \mathrm{~g} / \mathrm{cm} * 3$, therefore

the radiation dose in $\mathrm{Pb}-\mathrm{G}$ is actually $20 \%$ higher than measured by the propane-filled monitors.

For concrete $\mathrm{dE} / \mathrm{dx}=3.7 \mathrm{Mev} / \mathrm{cm}$ and interaction length $=40 \mathrm{~cm}$

For steel 1 interaction length $=16.8 \mathrm{~cm}$ 


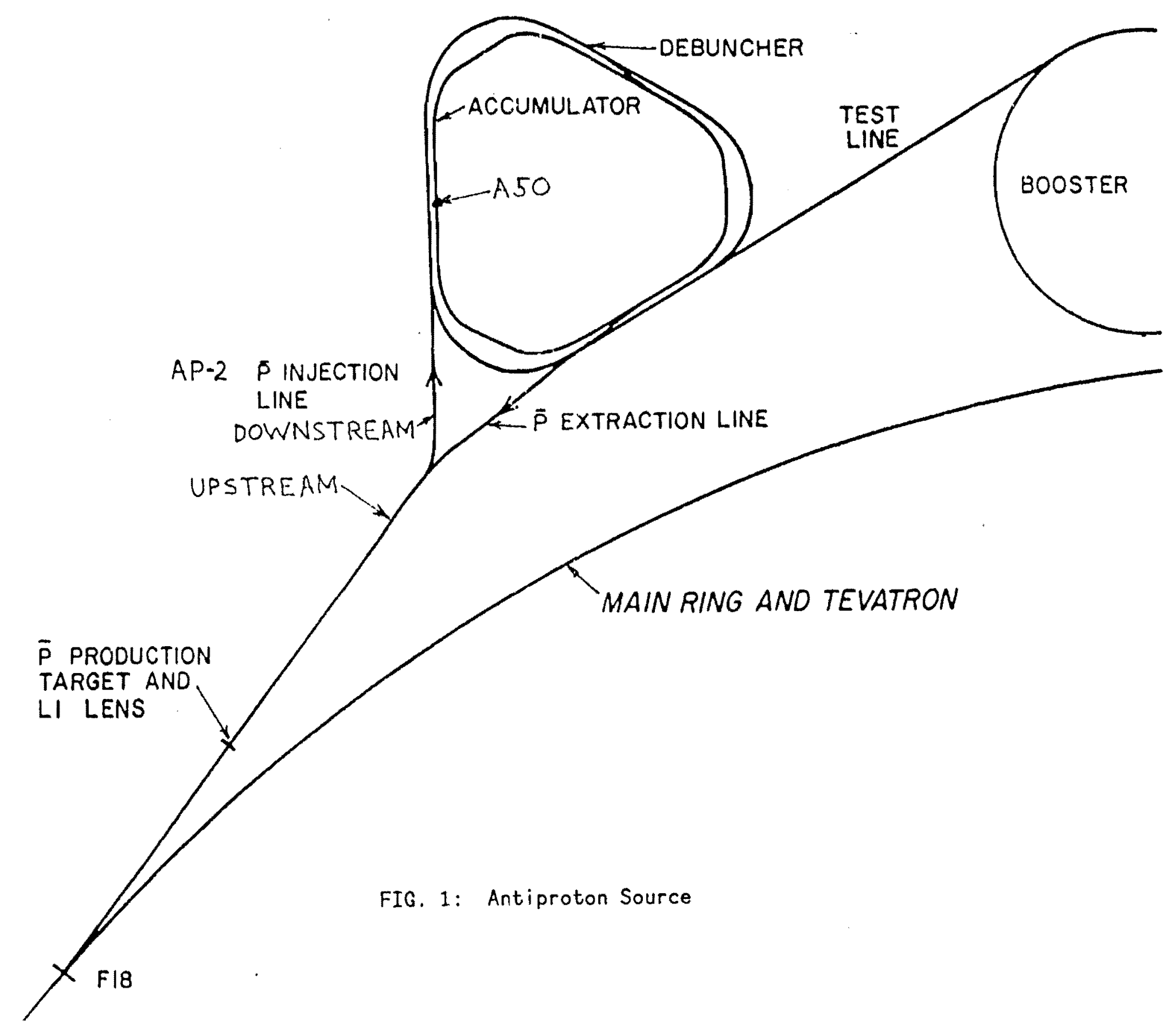




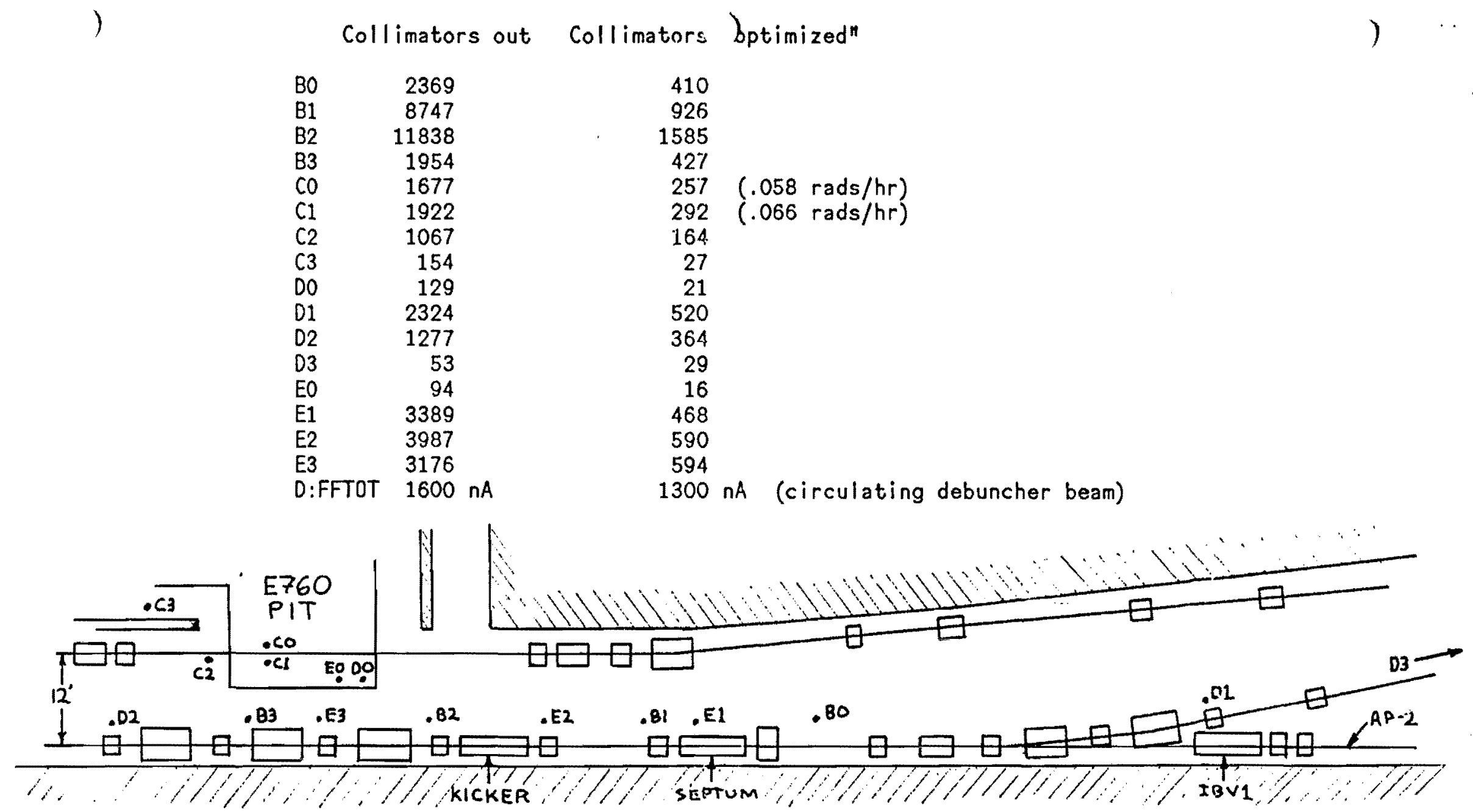

FIG. 2: Location of Radiation Monitors, 6/89: $\mathrm{CO}$ is at ceiling height; EO, DO on E760 pit floor; all others at beam height; DO is neutron monitor 


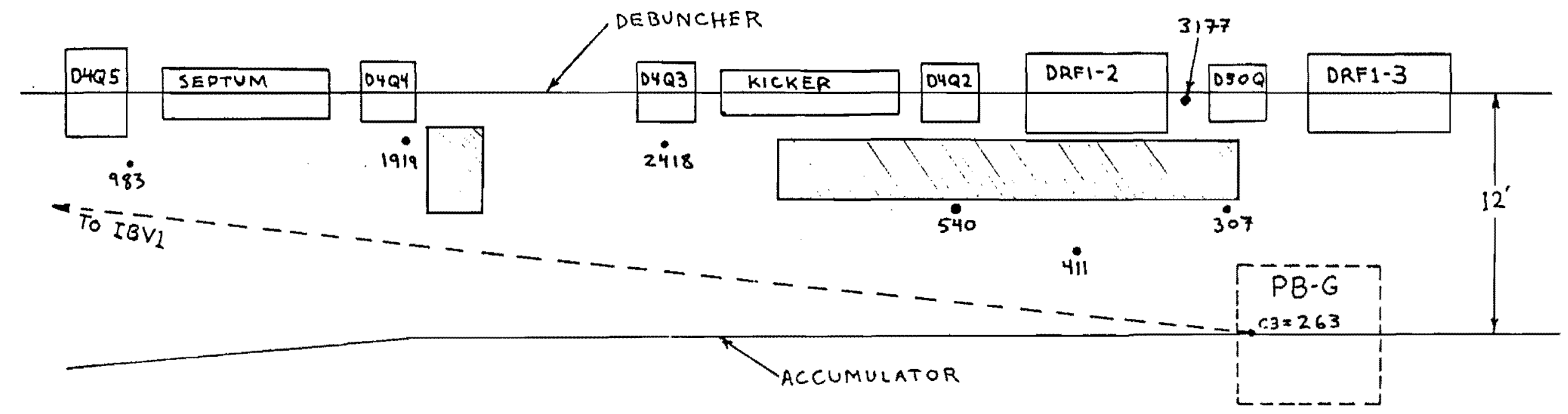

FIG 3a: Shielding location for configuration 3; monitor count rates are in cnts/supercycle, 19 ' 29 ' cycles/supercycle;

D:FFTTOT (circulating debuncher beam) not recorded 


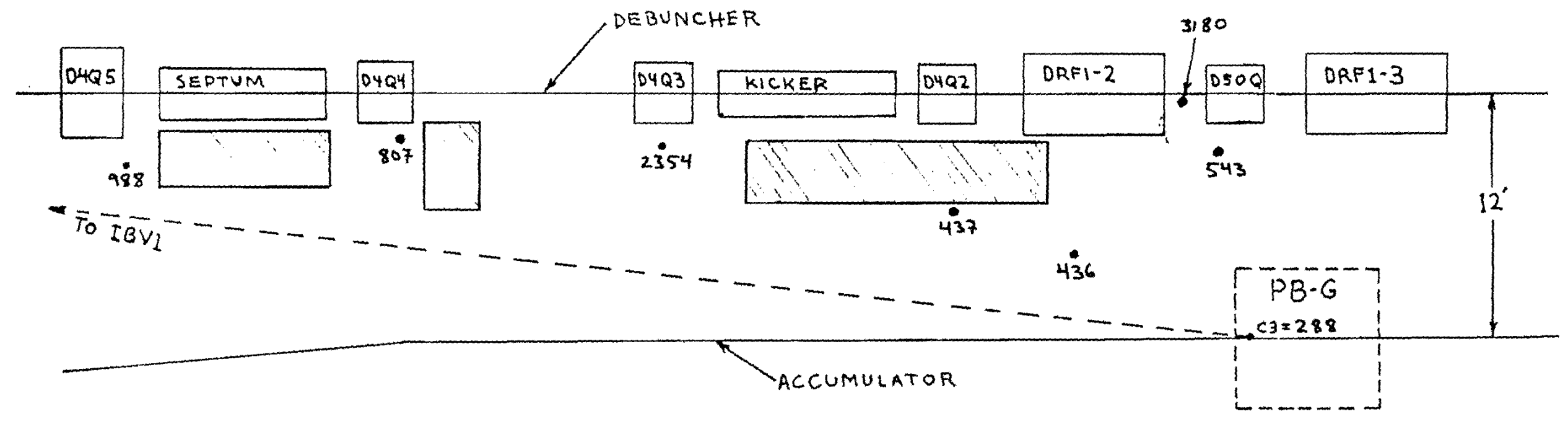

FIG 3b: Shielding location for configuration 4; monitor count rates are in cnts/supercycle, 19 ' $29^{\prime}$ cycles/supercycle; D:FFTTOT $=1300 \mathrm{nA} \quad 6 / 89$ 


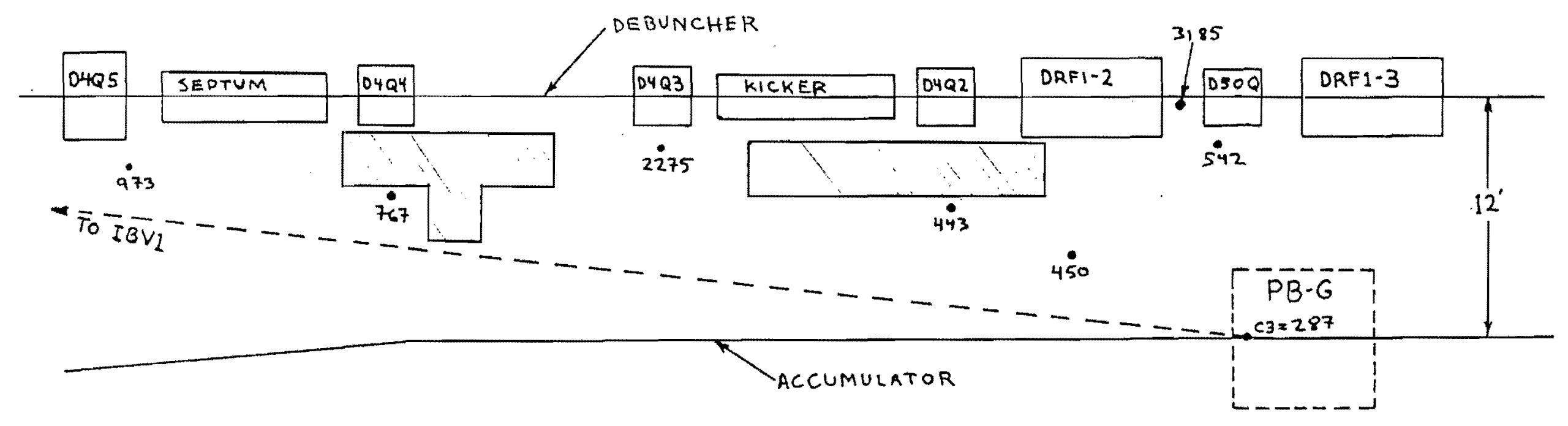

FIG 3c: Shielding location for configuration 5; monitor count rates are in cnts/supercycle, normalized to 19 ' 29 ' cycles/supercycle; D:FFTTOT (circulating debuncher beam) not recorded $6 / 89$ 


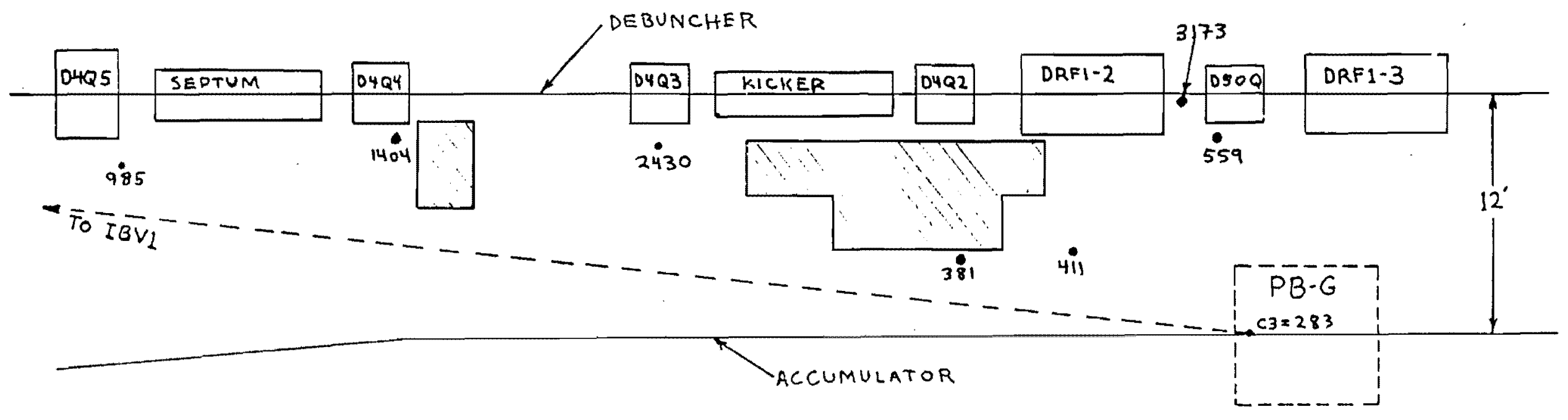

FIG 3d: Shielding location for configuration 6; monitor count rates are in cnts/supercycle, normalized to 19 ' $29^{\prime}$ cycles/supercycle; $D: F F T T O T=1400 \mathrm{nA} \quad 6 / 89$ 


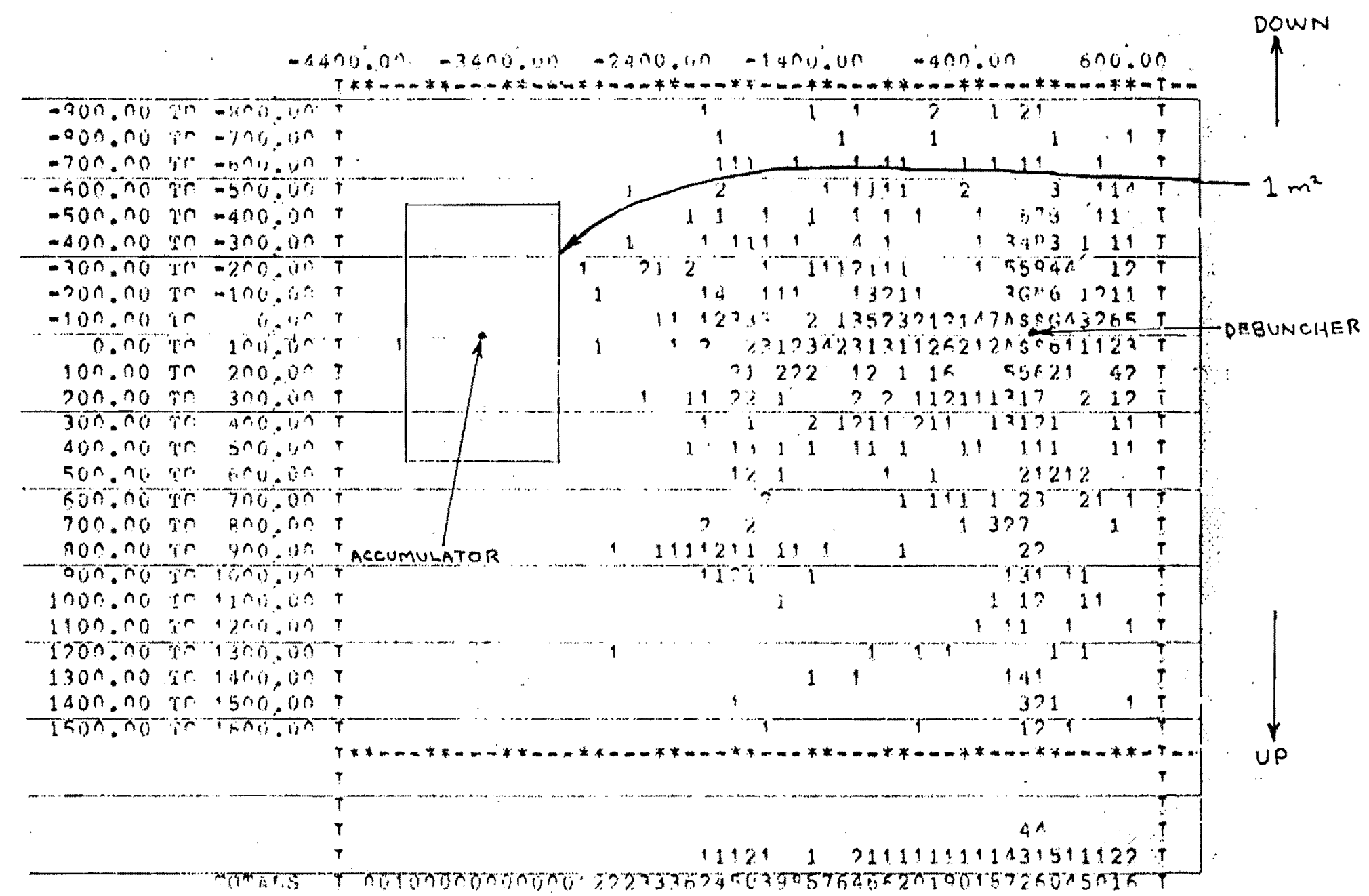

FIG. 4: Cross section of Accumulator enclosure at A50 with monte carlo data; horizontal and vertical scales are in $\mathrm{mm}$ and the vertical scale is upside down; each entry in the scatter plot is 1 muon; normalized to a $1 \mathrm{~mA} / \mathrm{hr}$ stack rate, 1 entry corresponds to $3 * 10 * *-4 \mathrm{rads} / \mathrm{hr}$ 


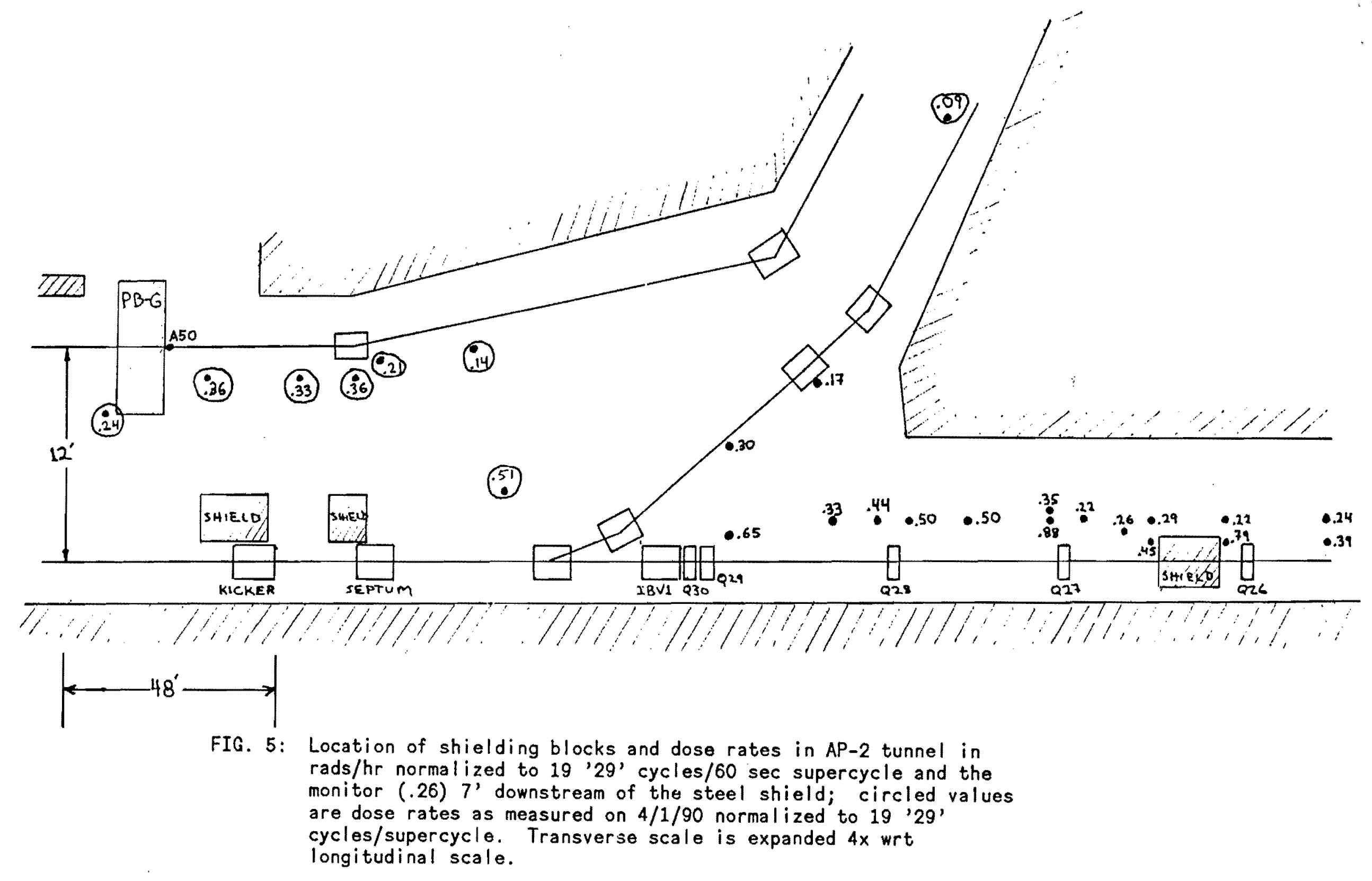




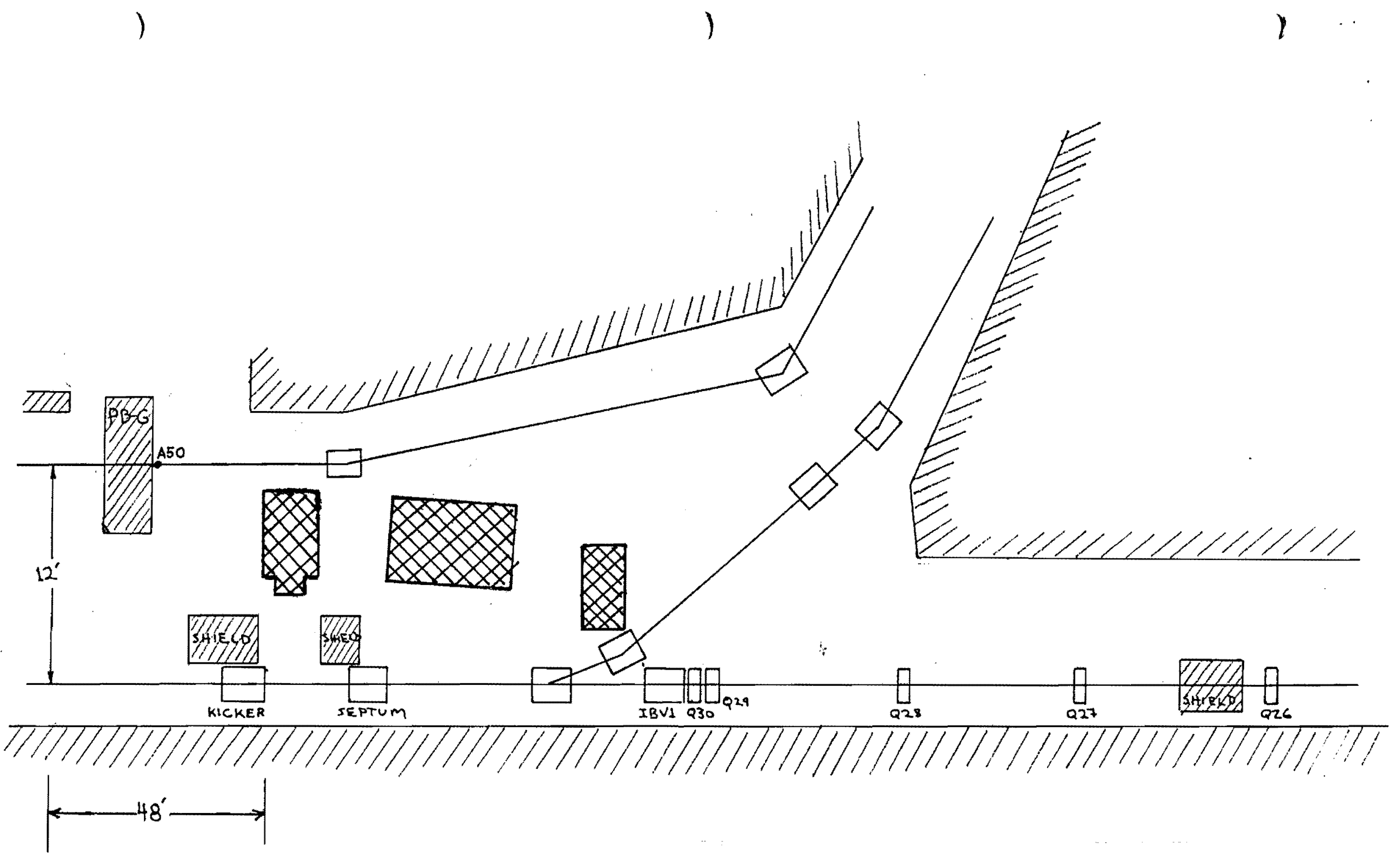

FIG. 6: Hatched areas show location of concrete shielding added on 12/90. Shielding is $6^{\prime}$ high. 
